\title{
Trajectory optimization by a direct descent process
}
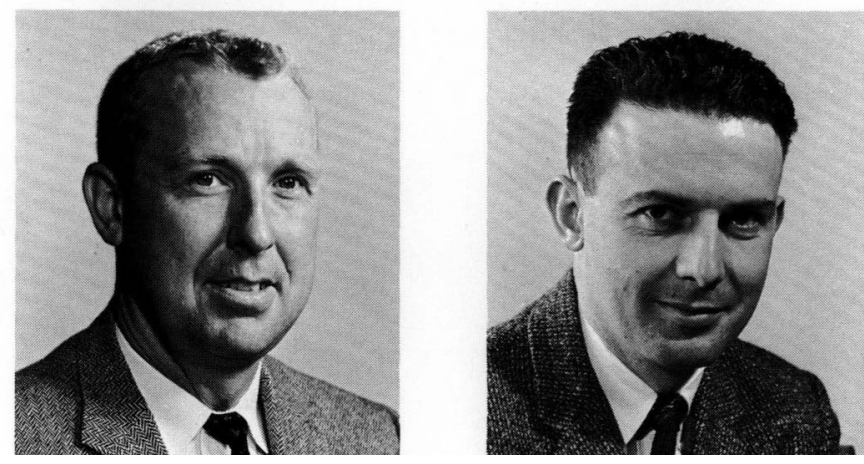

LAURENCE E. FOGARTY was born in Minnesota in 1920 and grew up near Bozeman, Montana where he graduated from Montana State College in 1940 with a degree in electrical "engineering. That same year he joined NACA (the forerunner of NASA) and worked as a wind-tunnel engineer at Langley Field, Virginia until 1944. In 1944 he joined the Navy and was stationed at the Taylor Model Basin, Washington, D.C., as a wind-tunnel engineer, where he remained after discharge from the Navy until 1947. In 1950 he received a PhD from Cornell University in aeronautical engineering, and after a short stay at North American Aviation, joined Link Aviation in Binghamton, New York. At Link he was concerned with flight simulation as supervisor of the Flight Mechanics Department and later as Chief Engineer.

In 1959 he joined the University of Michigan as Associate Professor in Aerospace Engineering. He also joined the founders of Applied Dynamics, serving as a vice-president of $A D I$ in '63-64. In 1965 he returned to the University of Michigan as a lecturer and research associate, and is currently Professor of Aerospace Engineering and Director of the University of Michigan Simulation Center.

He has served as a consultant to Link since 1959 and to Applied Dynamics since 1965. His major interests are in flight simulation, space vehicle mechanics, and analog computer applications.

$\mathrm{He}$ is an Associate Fellow of the AIAA, a member of IEEE and of $\mathrm{SCi}$, and Chairman of the SCi Flight Simulation Committee.

ROBERT M. HOWE was born in Oberlin, Ohio in 1925. He received his BS in Electrical Engineering from California Institute of Technology in 1945, his AB in Physics from Oberlin College in '47, the MS in Physics from the University of Michigan in '48, and his PhD from Mssachusetts Institute of Technology in ' 50.

Since 1950 he has been at the University of Michigan, where he is Professor of Aerospace Engineering and serves as chairman of the Department of Aerospace Engineering. His technical areas of teaching and research include design and application of analog computers, automatic control, and flight simulation. He has numerous publications in these areas, including a book on analog computers.

He was one of the founders of Applied Dynamics, Inc., and currently serves as board chairman. He was also a "founder" and first chairman of the Midwestern Simulation Council, one of our largest and most active regional Councils.

Dr. Howe is a member of Tau Beta Pi, Phi Beta Kappa, Sigma $X i$, and Simulation Councils Inc.

\author{
by L. E. FOGARTY and R. M. HOWE \\ University of Michigan \\ Aerospace Engineering Department \\ Ann Arbor, Michigan
}

This research was supported under NASA contract no. NASr 54(06). Reprinted with permission of the authors and Pergamon Press, the copyright owner.

\section{ABSTRACT}

The problem considered is that of trajectory optimization using step-by-step descent to minimum cost along the direction of the cost gradient with respect to the control. Using a hybrid computer, the gradient is computed directly as the response to nearly impulsive control perturbations. A method is presented for computing the gradient when several terminal constraints are enforced. Examples of application of the method are presented. It is concluded that the direct gradient computation method has some significant advantages over other methods.

\section{INTRODUCTION}

Computer methods of solution of variational problems are of considerable interest because only the simplest idealized problems can be treated analytically. The variational problem of space vehicle trajectory optimization has received much attention because of the relatively large increase of pay load in orbit due to even a small percentage decrease of fuel required.

A practical computer procedure for trajectory optimization is iterative in nature, giving a step-by-step approach to the optimal control program. The steps are taken in the direction of steepest descent, along the function-space gradient of the cost with respect to the control. Determination of the gradient is the major computer problem; if this can be accomplished rapidly and economically, a practically optimal control usually can be found easily.

The difficulty of computing the descent direction increases greatly when the control is restricted by the requirement that the trajectory must satisfy a set of terminal conditions or constraints. Imposition of terminal constraints changes the steepest descent direction from that of the cost gradient to the direction determined by an appropriate linear combination of the cost gradient and the gradients of all of the constraints with respect to the control. Thus the cost gradient and each of the constraint gradients must be computed, and, in addition, auxiliary computation must be performed to determine the coefficient multipliers of the constraint gradients. 
The function-space gradients of the cost and the terminal constraints with respect to the control also may be regarded as the responses at the terminal time to unit impulsive changes of the control which were applied at earlier times. High-speed iterative analog and hybrid computation methods which have recently become available permit one to introduce directly an approximatelyimpulsive change of control and determine the resulting change of the cost and any other terminal quantities, all simultaneously. This paper is concerned with a very direct, simple method of determining the cost and constraint gradients and with methods of using the resulting information to obtain an optimal control program.

\section{STATEMENT OF THE PROBLEM}

We are concerned with variational problems of the Mayer type:

a. The cost, /, to be minimized by selecting the best control or driving function, $\alpha(t)$, depends only on the terminal values of the state variables, $\left.x_{i}\right|_{t=t_{F}}$ and the terminal time, $t_{F}$ :

where

$$
J=J\left(x_{i F}, t_{F}\right) \quad i=1 \ldots n
$$

$$
x_{i F}=\left.x_{i}(t)\right|_{t=t_{F}}
$$

b. The state variables must satisfy the equations of motion, which usually are nonlinear.

$$
\left.\begin{array}{rl}
x_{i} & =f_{i}\left(x_{1}, x_{2}, \ldots x_{n}, \alpha, t\right) \quad 0 \leq t \leq t_{F} \\
x_{i}(0) & =x_{i 0} \\
i & =1 \ldots n
\end{array}\right\}
$$

c. There may be a number of terminal constraints which must be satisfied:

$$
\phi_{k}\left(x_{\left.i F^{r}, t_{F}\right)}=0 \quad k==1 \ldots m<n\right.
$$

A simple example of the type of problem considered is the celebrated "brachistochrone" problem which may be formulated as follows:

Determine the shape of a wire down which a bead will slide, without friction, from the origin to $x_{c}, y_{c}$ in least time. The path shape is specified by the path angle from the horizontal as a function of time, $\alpha(t)$.

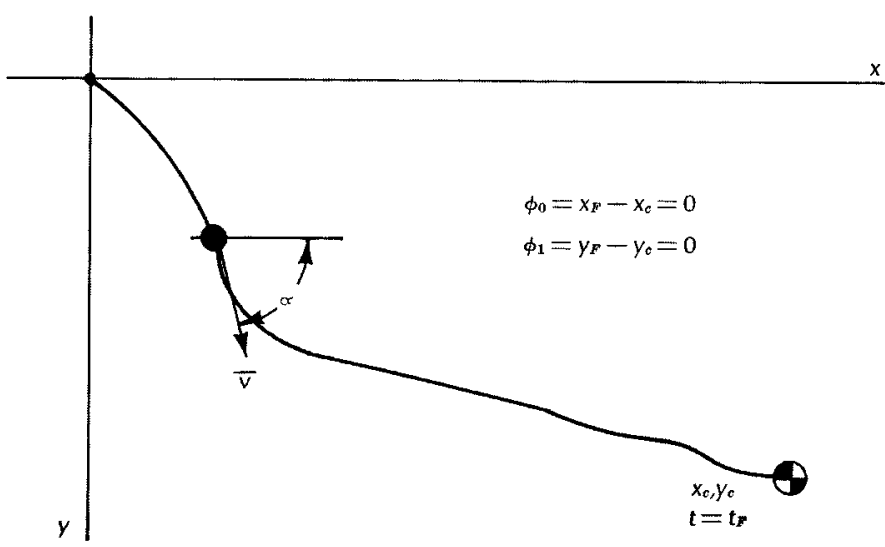

Figure 1-Brachistochrone problem
In this case the equations of motion are:

$$
\left.\begin{array}{l}
\dot{x}_{1}=\dot{v}=g \sin \alpha=f_{1}\left(x_{1}, x_{2}, x_{3}, \alpha\right) \\
\dot{x}_{2}=\dot{x}=x_{1} \cos \alpha=f_{2}\left(x_{1}, x_{2}, x_{3}, \alpha\right) \\
\dot{x}_{3}=\dot{y}=x_{1} \sin \alpha=f_{3}\left(x_{1}, x_{2}, x_{3}, \alpha\right)
\end{array}\right\}
$$

The angle of the wire to the horizontal is the control or driving function, $\alpha(t)$. The terminal constraints are:

$$
\left.\begin{array}{l}
\Phi_{0}=x_{F}-x_{c}=0 \\
\Phi_{1}=y_{F}-y_{c}=0
\end{array}\right\}
$$

The cost, which is to be minimized, is just the terminal time:

$$
J\left(x_{i F}, t_{F}\right)=t_{F}
$$

Problems of the Mayer type have been treated extensively, using gradient methods, by Kelley ${ }^{1}$, Bryson, Carroll, Mikami, and Denham, Wingrove and Raby", and others. These investigators have used two entirely different methods to determine the function-space gradient of the cost with respect to the control. Kelley, Bryson, et al., have used indirect adjoint equation methods, whereas Wingrove and Raby measured the gradient directly as the response to an "impulsive" change of control.

The object of the present investigation is to develop the direct gradient method, using procedures related to those of Wingrove and Raby. In particular, we wish to show how the impulse response procedure may be implemented on a high-speed iterative differential analyzer to obtain a great amount of information about the effect of the control variable on the cost and the constraints. This information is readily usable to determine a practical optimum trajectory.

The general gradient computation procedure used here in minimizing the performance index, $J$, consists of the following steps:

a. Determine, by any means, an initial or nominal program of the driving function, $\bar{\alpha}(t)$, which satisfies the terminal constraints, but does not necessarily minimize the cost, $)$.

b. Solve the equations of motion using $\bar{\alpha}(t)$ and determine the cost of using this program, $J_{0}$.

c. Add an approximately impulsive change, $K \delta_{\alpha i}$ to $\bar{\alpha}$ so that $\alpha=\bar{\alpha}+K \delta_{\alpha i}$. Here $\delta_{\alpha i}$ means an approximation of $\delta\left(t-t_{i}\right)$, the delta function or unit impulse. That is, $\delta_{\alpha i}$ is an approximately impulsive unit change of $\alpha$ located "at" $t=t_{i}$. For this investigation we used a triangular shaped "impulse," $\delta_{\alpha i}$, shown in figure 2 .

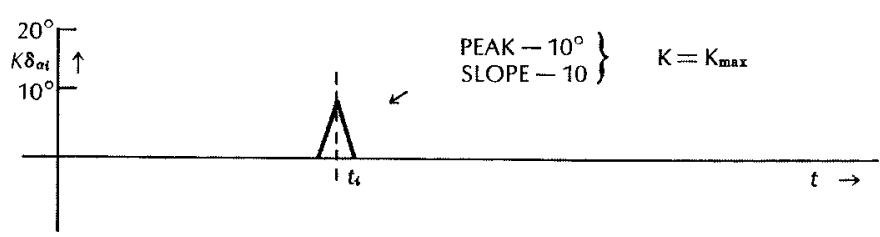

Figure 2-"Impulsive" control perturbation 
d. Measure the change of / due to $K \delta_{\alpha i}$ and plot against the location of the impulse, $t_{i}$. The cost response $\delta /$ to $K \delta_{\alpha i}$ is approximately proportional to the impulse response, or weighting function, of the cost with respect to $\alpha$ which we will call $W_{a}{ }^{J} . W_{a}{ }^{J}$ may be viewed as the function-space gradient of the cost with respect to the control or driving function.

e. Simultaneously with (d), measure and plot the impulse responses of any terminal constraints, $\phi_{k}$. Call these $W_{\alpha}^{\phi k}(k=1 \ldots m)$. Note that $W_{\alpha}{ }^{J}$ and all $W_{\alpha}{ }^{\phi k}$ can be determined simultaneously on a single computer run.

$f$. With the gradient of the cost and that of each of the terminal constraints known, several different "steepest descent" procedures can be used to change the control, $\alpha(t)$, so that the new cost will be less than the old and the terminal constraints still will be satisfied. The procedure selected is repeated until no further improvement of $J$ is obtained, indicating that $J$ is stationary and should be checked to see if a minimum has been reached.

Some of these procedures are discussed in detail in the examples presented.

Since it was desired to accomplish steps (b), (c), and (d) many times each second (20 to 50 times per second), most operations were accomplished automatically by means of the control features of the iterative differential analyzer.

\section{ITERATIVE DIFFERENTIAL ANALYZER}

The iterative differential analyzer used was a small (48 amplifier) analog computer with 12 integrators, each with individually controllable mode switching, and a small complement of parallel, patchable, asynchronous logic elements. See figure 3. Two problem patchboards are used, one for the analog signals and one for logic signals. The equations to be solved are patched on the analog board, and the control scheme is patched on the logic board. All control is by means of logic signals: logic " 0 " $(-6 \mathrm{~V})$ and logic " 1 " (0V). All communication between the analog and logic elements is by means of logic level signals.

The logic complement consists of 18 OR gates, 14 flipflops, 4 variable pulsers (one-shot multivibrators) and one 4-cell shift register. The output of each logic element and

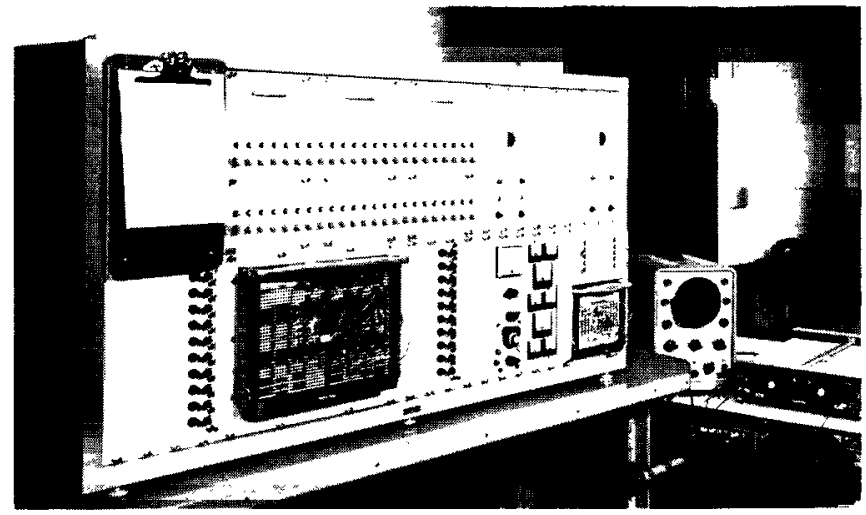

Figure 3-Iterative differential analyzer

the complement of the output are terminated on the logic board, so that OR, AND, NOR and NAND operations can be performed. Logic level signals operate the integrator mode controls and four free high-speed switches, providing analog operations under control of the logic elements.

Communication from the analog board to the logic board is by means of logic signal outputs from four comparators. Comparator inputs are on the analog board; their outputs are on the logic board. When the sum of the input voltages to a comparator is greater than zero, the output on the logic board is logic 0; otherwise it is logic 1. A small amount of hysteresis is provided to suppress noise.

\section{THE ITERATIVE CONTROL SCHEME}

The control scheme used in determining the impulse responses applies to the general problem and is shown in the block diagram, figure 4 . The equations of motion, cost computation, and terminal constraint computation are peculiar to a given problem and are discussed in the examples. The method of using the gradient information in the steepest descent procedure and the method of handling terminal constraints also are discussed separately.

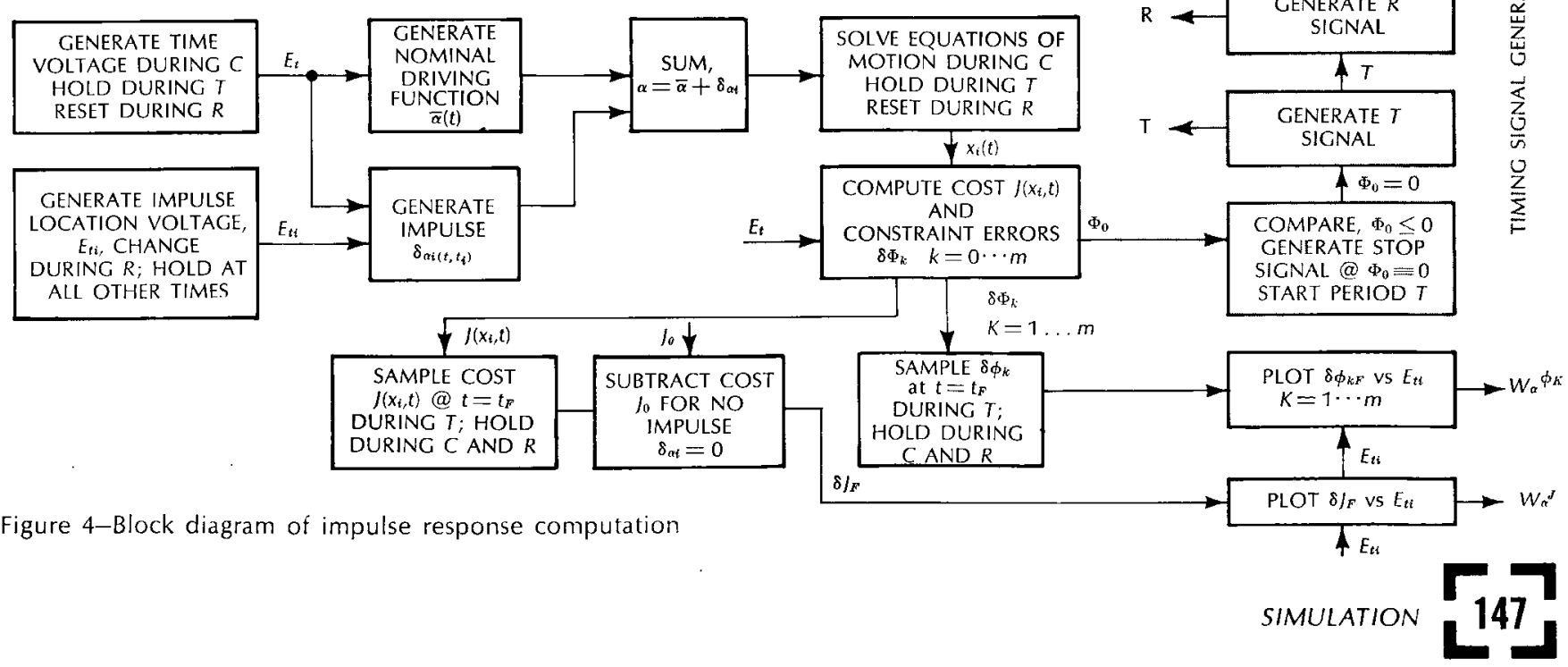


There are three periods during one computation cycle; we will call them $C, T$, and $R$. The $C$ (compute) period runs from the start of solution of the equations of motion until a stopping condition is reached at $t=t_{F}$. The $T$ (track) period runs from $t=t_{F}$ until sufficient time for memory units (track and hold amplifiers here) to have accepted the values to be held. At the end of the $T$ period, the track and hold units are placed in the "Hold" mode, and then the trajectory integrators are placed in the "Reset" or "IC" mode. The $R$ (reset) period lasts sufficient time for the integrators to accept the initial conditions, for the impulse $K \delta_{\alpha i}$ to be advanced to a new location, $t_{i+1}$, and for any other parameter changes to be made, after which a new $C$ period starts. The three periods, $C, T, R$ are determined by a three period "clock," shown in figure 5 . The end of the $C$ period is determined by the occurrence of a stopping event: either $t=t_{F}$, if $t_{F}$ is fixed, or $\Phi_{0}\left(x_{F}, t_{F}\right)=0$. When the stopping event occurs, a pulser, $P_{1}$, is triggered causing the output of $P_{1}$ to be logic one for a preset time period, which is the $T$ period. When $P_{1}$ output drops to logic zero at the end of the $T$ period, $P_{2}$ is triggered to start the $R$ period, and when $P_{2}$ output drops to zero, the next $C$ period starts. (As shown in figure 5 , a short delay between the end of the $T$ period and start of the Reset period is generated by pulser $P_{3}$ to ensure that the track-and-hold amplifiers are holding before the integrator Reset mode is

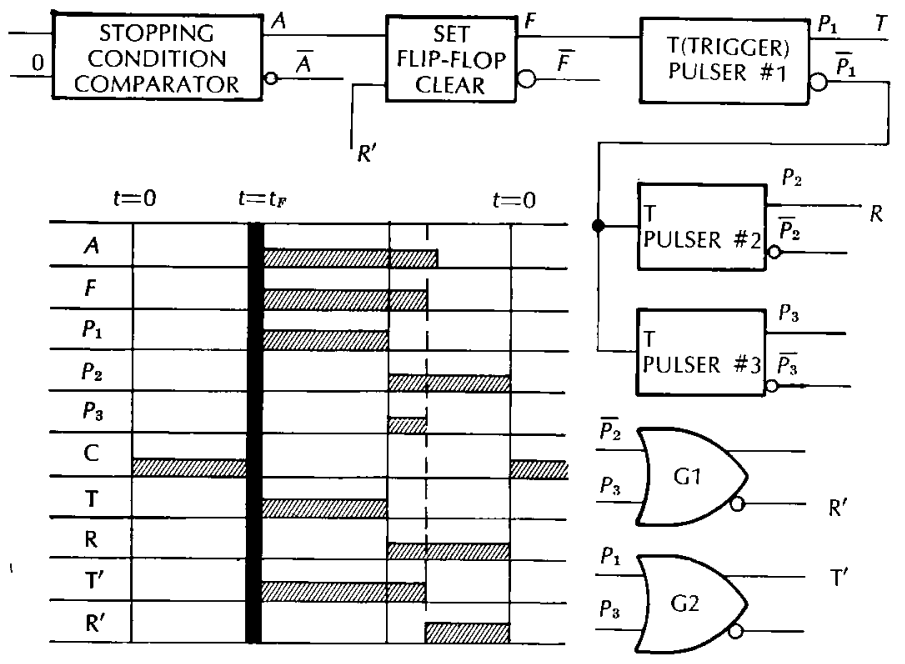

Figure 5-Timing signal generator started.) The control signals are obtained by combining suitably the $C, T, R$ signals from the clock. In addition, master control signals are introduced to start the computation, to interrupt it during a sequence of iterations, and to initialize all elements in preparation for a new run.

The impulsive change of control, $\delta_{\alpha i}$, is generated by the simple diode circuit shown in figure 6 . The time location of the impulse, $t_{i}$, is controlled by the voltage, $E_{t i}$, the output of an integrator with a small constant input. During the $R$ period, while the equations of motion are being initialized, the $E_{t i}$ integrator is in the "Operate" mode; it is in the "Hold" mode at all other times during a run. Thus the $E_{t i}$ voltage is increased by a small constant amount before each computation step, automatically advancing the impulse location, $t_{i}$, in small equal increments from 0 to $t_{F}$ during a complete run.

\section{EXAMPLE 1: BRACHISTOCHRONE PROBLEM}

As a first demonstration of the direct gradient measurement method, consider the brachistochrone problem mentioned earlier, but with a single terminal constraint, $x_{F}=1$. That is, determine the shape of the wire down which a bead will slide without friction, from the origin to a wall located at $x=x_{c}$, in least time.

The control or driving function, $\alpha(t)$, is the angle of the wire to the horizontal, the cost is the terminal time itself, $J=t_{F}$, and there is one terminal constraint, $\phi_{0}=\left(x_{F}-1\right)$ $=0$.

When the terminal time is included in the cost and is therefore variable, as in this case, some condition or event other than $t=t_{F}$ must be used to stop the computation. In this case, the obvious choice is the occurrence of $x=x_{c}$. Thus the voltage $x_{c}-x$ is used as the input to the stoppingcondition comparator in figures 4 and 5 . The logic signal output of the comparator terminates the $C$ period and starts the $T$ period. An integrator with a constant input gives a voltage proportional to time, $E_{t}$; this voltage at $t=t_{F}$, i.e., when $x=x_{c}$, is the input to the "Sample Cost" block in figure 4 (a track-and-hold amplifier). For this simple case, the only terminal constraint is used as a stopping condition, and every solution therefore satisfies the constraint. It therefore is unnecessary to measure any constraint gradients, $W_{\alpha}{ }^{\phi t}$.

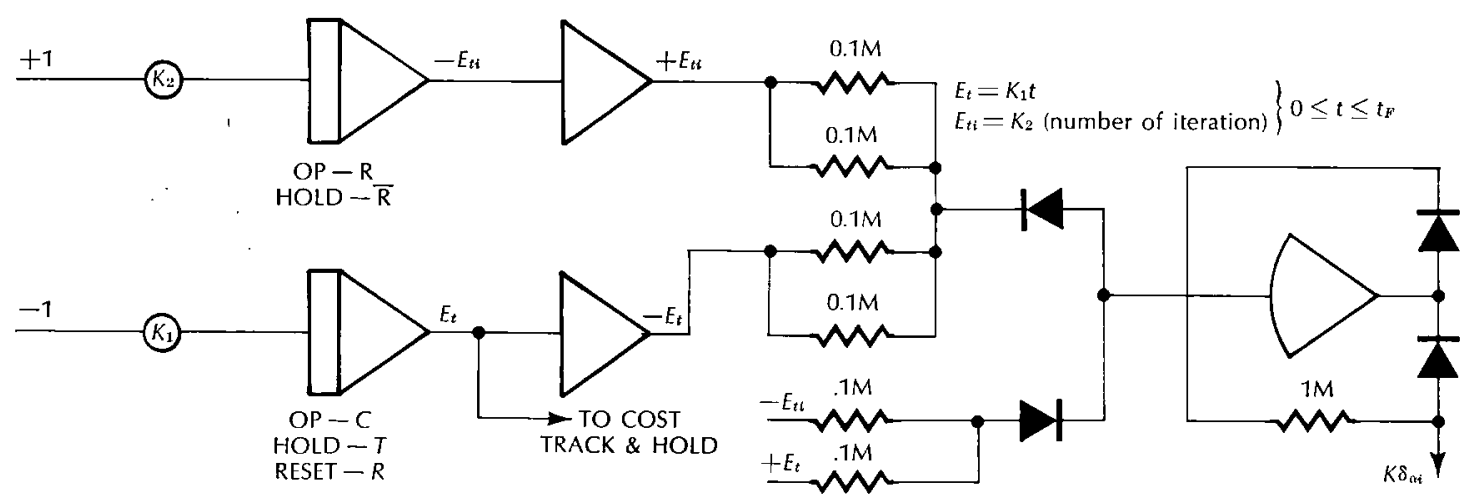

Figure 6-Diode circuit for generating control perturbation, $K \delta_{a i}$ 


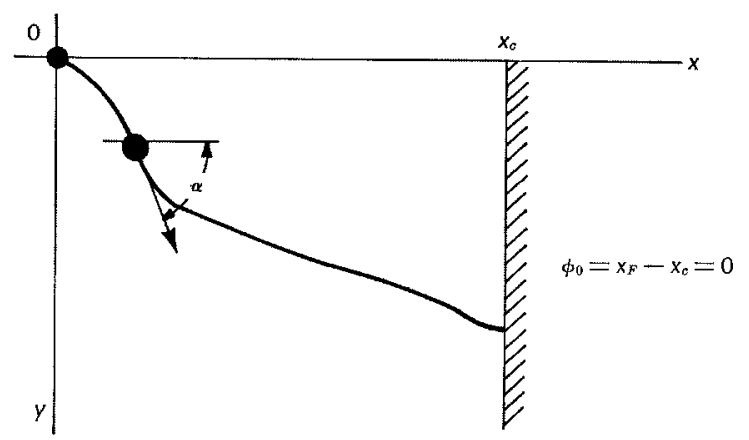

Figure 7-Brachistochrone example

We chose as a first nominal trajectory $\alpha=$ constant $=$ $45^{\circ}$ and measured the gradient of the terminal time, $W_{\alpha}{ }^{J}$, about $\alpha=45^{\circ}$. Figure 8 shows the plot (taken directly from the xy recorder) of $W_{\alpha}^{J} v s . t_{i}$, where it will be recalled that $t_{i}$ is the time of application of the control impulse, $K \delta_{\alpha i}$. All of the points shown were computed and plotted in about 20 seconds - the speed limitation was the $x y$ plotter. The gradient in this simple case can be determined analytically to be proportional to time, which checks within the accuracy of the plotter.

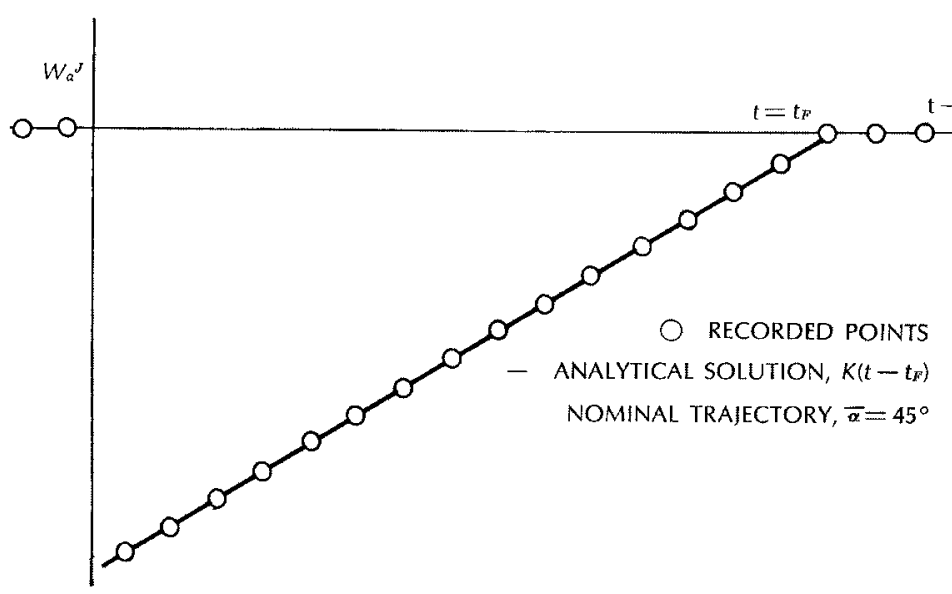

Figure 8-Response of terminal time, brachistochrone, one terminal constraint used as stopping condition

The usual steepest descent procedure is to generate a new control program, $\overline{\alpha_{1}}(t)$, with

$$
\overline{\alpha_{1}}=\bar{\alpha}+\delta \alpha=\bar{\alpha}-K W_{\alpha}^{J}
$$

The constant, $K$, is selected to produce a desired achievable amount of improvement of $/$.

In this case, since $W_{\alpha}{ }^{J}$ is linear in time, $\overline{\alpha_{1}}=a-b t$, and the constants $a$ and $b$ were simply adjusted by hand until $t_{F}$ appeared to be minimized. (Note that these are just the first steps of the Rayleigh-Ritz procedure, with the assumption that $\alpha(t)$ can be expanded in a power series.) Because of the high iteration rate, the apparent minimum was reached in only a few trial settings of $a$ and $b$. Figure 9 shows the final control program, with maximum positive and negative control perturbations superimposed. Figure

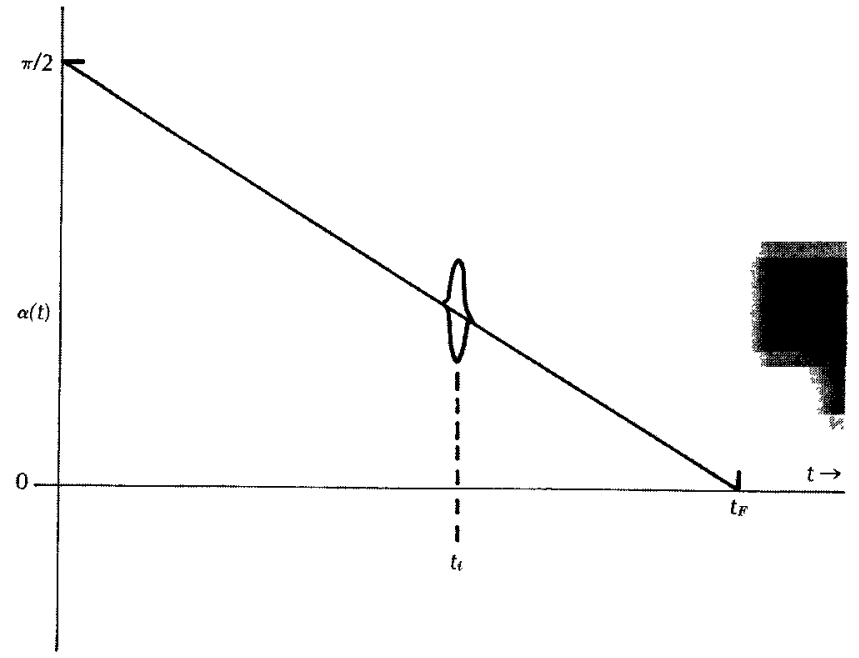

Figure 9-Optimum driving function program, brachistochrone with maximum positive and negative "impulsive" change

10 shows the trajectory resulting from the best settings of $a$ and $b$, and also shows the effect on the trajectory of the maximum control perturbations that were used.

Because it was known that the control program achieved above was near the desired optimal program, it was tested for optimality as follows: at a number of times, $t_{i}$, the $\mathrm{im}$ pulse response, $\delta /$ was plotted against the size (area) of the impulse. That is, instead of using a fixed impulse size, $K \delta_{\alpha i}$ (where $K$ is fixed) and advancing the location of $\delta_{\alpha i}$ at each iteration, the location, $t_{i}$, was fixed for a series of iterations, with each iteration corresponding to a different impulse area, $K$. At each $t_{i}$, the value of $\delta /$ was plotted against the area of the impulse, resulting in a plot from

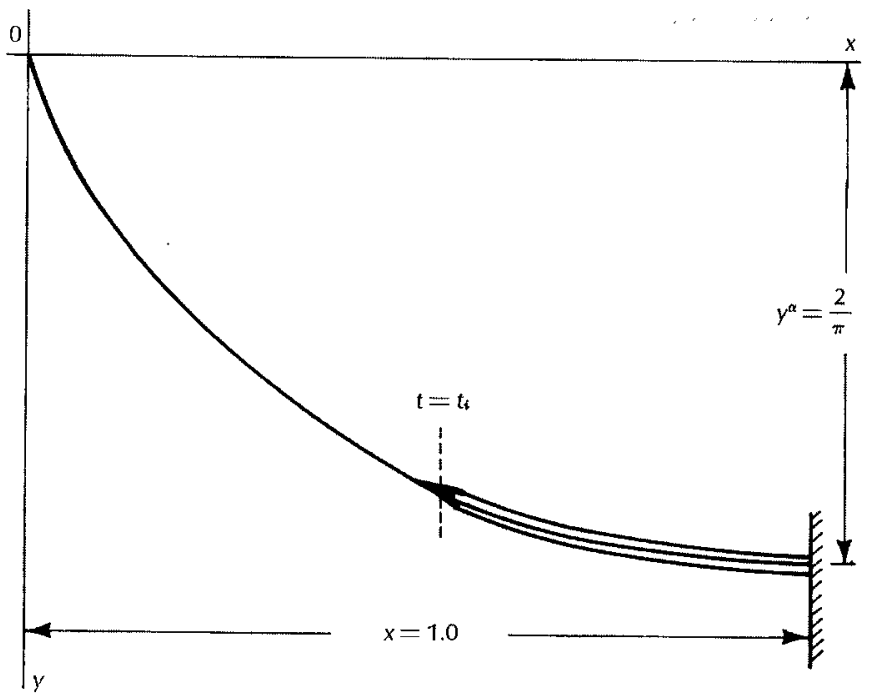

Figure 10--Brachistochrone, showing effect of maximum positive and negative impulses in $\alpha$ 
which the second variation could be determined. Figure 11 shows the results of this procedure. It is seen that the gradient does indeed vanish, within the accuracy of measurement, indicating that the best control has been achieved. The final trajectory was compared with the known analytical solution (a cycloid) and was found to agree within a few percent.

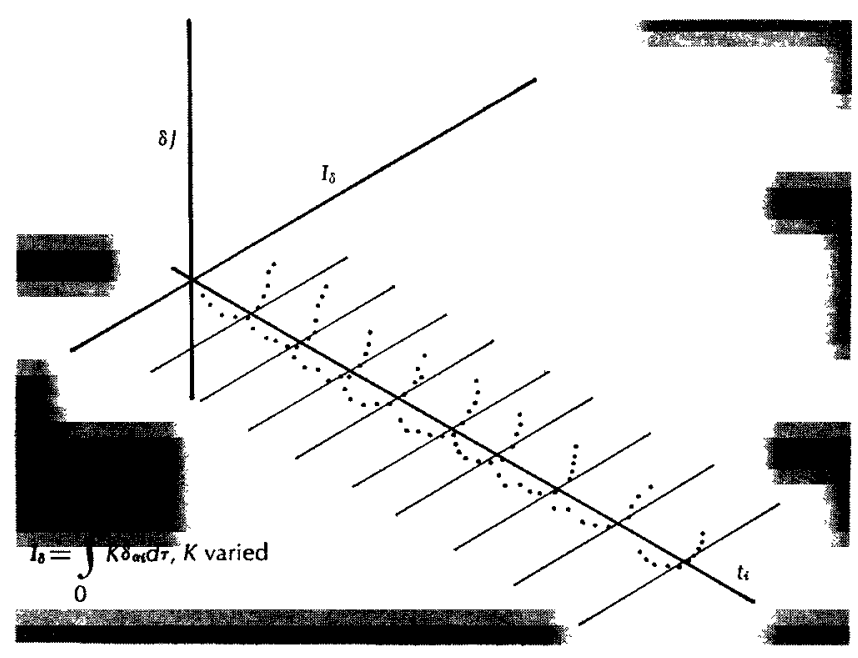

Figure 11-Plots of $\delta / \mathrm{vs}$. Io for various $t_{i}, \alpha$ optimal. Brachistochrone problem

\section{TERMINAL CONSTRAINTS}

When there are no terminal constraints, or if the only constraint is used as a stopping condition as in the previous example, the descent to the minimum cost normally proceeds step-by-step in proportion to the measured gradient, since this is the direction of steepest descent. If additional terminal constraints are imposed (for example if the terminal value of $y$ is specified instead of being left free in the brachistochrone problem), then it is apparent that the direction of descent generally must be changed to avoid violating the constraints.

For simplicity, consider the case of a single terminal constraint, $\phi_{1}\left(x_{i}\right)=0$, and let the terminal time be fixed. As before, let the impulse response of the cost with respect to the control be $W_{c}^{J}$ and the impulse response of the constraint be $W_{\alpha}{ }^{\phi}$. If a descent step is taken in the direction of the cost gradient, without regard for the constraint, so that $\delta \alpha_{I}=K^{J} W_{n}^{J}$, the cost will be decreased, but, naturally, the constraint $\phi_{1}=0$ will be violated. To first order, the constraint error, $\delta \phi_{1}$ will be:

$$
\delta \phi_{1}=\int_{0}^{t_{\boldsymbol{F}}} K^{J} W_{\alpha}{ }^{J} W_{\alpha}{ }^{\phi} d \tau
$$

In order to correct the constraint error in the most efficient way, that is, to reduce the error to zero in the direction of steepest descent, we should add to the control function an increment

$$
\delta \alpha_{\phi}=K^{\phi} W_{\alpha}^{\phi}
$$

where $K^{\phi}$ is adjusted to give $\delta \phi_{1}=0$. Using this condition,

$$
\delta \phi_{1}=\int_{0}^{t_{F}}\left(K^{J} W_{\alpha}^{J} W_{\alpha}^{\phi}+K^{\phi} W_{\alpha}^{\phi} W_{\alpha}^{\phi}\right) d \tau=0
$$

from which:

$$
K^{\phi}=-\left(\frac{I_{J \phi}}{I_{\phi \phi}}\right) K^{J}
$$

we have used the notation:

$$
\left.\begin{array}{c}
I_{J \phi}=\int_{0}^{t_{F}} W_{\alpha}{ }^{J} W_{\alpha}{ }^{\phi} d \tau \\
I_{\phi \phi}=\int_{0}^{t_{F}}\left(W_{\alpha}{ }^{2}\right)^{2} d \tau
\end{array}\right\}
$$

Thus, with a single "free" terminal constraint, one should take

$$
\delta \alpha=K\left(W_{\alpha}^{J}-\frac{I_{J \phi}}{I_{\phi \phi}} W_{\alpha}^{\phi}\right)
$$

where $K$ determines the size of the descent step and the ratio $I_{J \phi} / I_{\phi \phi}$ is such that the constraint, $\phi_{1}=0$, is not violated, to first order. (If a constraint error existed at the end of the previous iteration, of course, this error would be eliminated, to first order, by taking $\delta \phi_{1} \neq 0$ in equation (11).) If more than one constraint is imposed, so that it is required that

$$
\phi_{k}=0 \quad k=1 \ldots m
$$

it can be shown that the descent steps should proceed in the direction of a linear combination of the impulse responses:

$$
\delta \alpha=K\left(W_{\alpha^{\prime}}{ }^{\prime}+\sum_{k=1}^{m} C_{k} W_{\alpha}^{\phi k}\right)
$$

The $C_{k}$ are detcrmined so that none of the constraints will be violated, to first order. Using the condition that all $\delta \phi_{l_{i}}=0$, it can be shown that

$$
C_{k}=\frac{\Delta k}{\Delta}
$$

where

$$
\Delta=\left|\begin{array}{llll}
I_{11} & I_{12} & \ldots \ldots \ldots & \ldots \\
I_{21} & I_{22} & \ldots \ldots \ldots & \ldots \\
I_{m 1} & \ldots & \ldots \ldots \ldots & I_{2 m} \\
I_{m m}
\end{array}\right|
$$

and $\Delta k$ is the same determinant as $\Delta$ except that the $k$ th column is replaced by

$$
\left|\begin{array}{c}
-I_{1 J} \\
-I_{2 J} \\
\cdot \\
\cdot \\
-I_{m J}
\end{array}\right|
$$


We have used the notation

$$
\left.\begin{array}{c}
I_{i j}=\int_{0}^{t_{F}} W_{\alpha}^{\phi i} W_{\alpha}^{\phi j} d \tau \\
I_{i J}=\int_{0}^{t_{F}} W_{\alpha}^{\phi i} W_{\alpha}^{J} d \tau
\end{array}\right\}
$$

It is apparent that this procedure, which is essentially that given by Bryson et al. ${ }^{2}$, substantially increases the computation load and can be expected to have an adverse effect on the convergence of the iterative steepest descent process.

Our procedure for handling terminal constraints was devised to utilize the high iteration rate of the differential analyzer in determining, by direct computation, the steepest descent direction shown in equation (16), orthogonal to the impulse responses of the terminal constraints.

For simplicity, consider first the case of a single terminal constraint which is not used as a stopping condition. Assume that the impulse response of the constraint has been measured as described earlier, so that $W_{\alpha}^{\phi}$ is known. Now consider the response of the cost to an incremental control function of time which is composed of a linear combination of a unit impulse and the constraint impulse response function:

$$
\delta \alpha=K \delta_{\alpha i}+D W_{\alpha}{ }^{\phi}(t)
$$

The multiplier, $D$, is to be chosen so that the constraint is satisfied, and $D$ therefore is a function of the location of the impulse, $t_{i}$. Denoting the response of the cost to $\delta \alpha$ with a superscript $\phi$, then, to first order:

$$
\delta J^{\phi}=\int_{0}^{t_{F}} W_{\alpha}^{J} \delta \alpha d \tau=\int_{0}^{t_{1}} W_{\alpha}^{J}\left(K \delta_{\alpha i}+D W_{\alpha}{ }^{\phi}\right) d \tau
$$

Using the properties of the unit impulse:

$$
\delta f^{\phi}=K W_{\alpha}^{J}\left(t_{i}\right)+D\left(t_{i}\right) I_{J \phi}
$$

Since $D$ is to be adjusted at each $t_{i}$ so that $\delta \phi=0$,

$$
\delta \phi=\int_{0}^{\mathrm{t} F} W_{\alpha}^{\phi} \delta \alpha d \tau=\int_{0}^{\mathrm{t} p} W_{\alpha}^{\phi}\left(K \delta_{\alpha i}+D W_{\alpha}^{\phi}\right) d \tau=0
$$

and therefore:

$$
D\left(t_{i}\right)=\frac{-K W_{\alpha}^{\phi}\left(t_{i}\right)}{I_{\phi \phi}}
$$

Using this value for $D\left(t_{i}\right)$, the response of the cost to the augmented impulse becomes:

$$
\delta J^{\phi}=K\left[W_{\alpha}^{J}\left(t_{i}\right)-\frac{I_{I \phi}}{I_{\phi \phi}} W_{\alpha}^{\phi}\left(t_{i}\right)\right]
$$

Comparing this expression with equation (14), we see that the response to the augmented impulse is in the direction of steepest descent, orthogonal to the constraint influence function.

When there are several terminal constraints to be satisfied simultaneously, it can be shown by a process similar to the preceding that

$$
\delta /^{\phi}=K\left[W_{\alpha}{ }^{J}\left(t_{i}\right)+\sum_{k=1}^{m} C_{k} W_{\alpha}^{\phi k}\left(t_{i}\right)\right]
$$

which agrees with equation (16). $\delta / \phi$ is the response to the augmented impulsive control perturbation:

$$
\delta \alpha=K \delta_{\kappa_{i}}+\sum_{k=1}^{m} D_{k} W_{\alpha}^{\phi k}
$$

The $D_{k}\left(t_{i}\right)$ are determined by the requirement that $\delta \phi_{k}$ $=0, k=1 \ldots \mathrm{m}$.

We modified our control program so that the response $\delta f^{\phi}$ could be computed as follows:

The constraint gradients, $W_{\alpha}{ }^{\phi k}$, were first computed all simultaneously, using the procedure shown in the block diagram, figure 4 . The functions $W_{a}{ }^{\phi k}(t)$ then were set on diode function generators and a simple iterative automatic parameter adjustment scheme was used to set the parameters, $D_{k}$, so that all $\delta \phi_{l i}$ were zero at each $t_{i}$.

There are a number of iterative parameter adjustment procedures which can be used to set the $D_{k}{ }^{4}$ Since the $W_{c k}^{\phi k}$ represent steepest descent directions for the $\delta \phi_{l}$, if the $\delta \phi_{k}$ and the adjustment steps of the $D_{k}$ are small enough, either a simultaneous or a sequential parameter adjustment procedure should converge. We therefore chose the simplest simultaneous adjustment scheme, represented by the block diagram shown in figure 12 . For a single constraint, the circuit gain can be adjusted so that any small error, $\delta \phi$, is removed to a good approximation in a single step. When there are several constraints which interact, more iterations are required at each $t_{i}$, but convergence should be obtained since the errors are reduced in the direction of steepest descent. Also note that (assuming the constraints to be linearly independent) an orthogonalization procedure could be used to minimize interaction. For the example presented here, it was found that the constraint errors were eliminated to a good approximation in four iterations. The control scheme therefore was modified to provide four adjustments of the $D_{k}$ at each $t_{i}$ before $\delta /^{\phi}$ was plotted and the impulse shifted to $t_{i+1}$. The four-bit shift register was used to count the iterations; the plotter signal and the signal to increment $E_{i}$ in figure 6 were taken from the fourth register stage. This procedure, of course, increased the actual computing time by a factor of four, but it still was negligible in comparison with other times required-for example, adjusting the hand-set function generators.

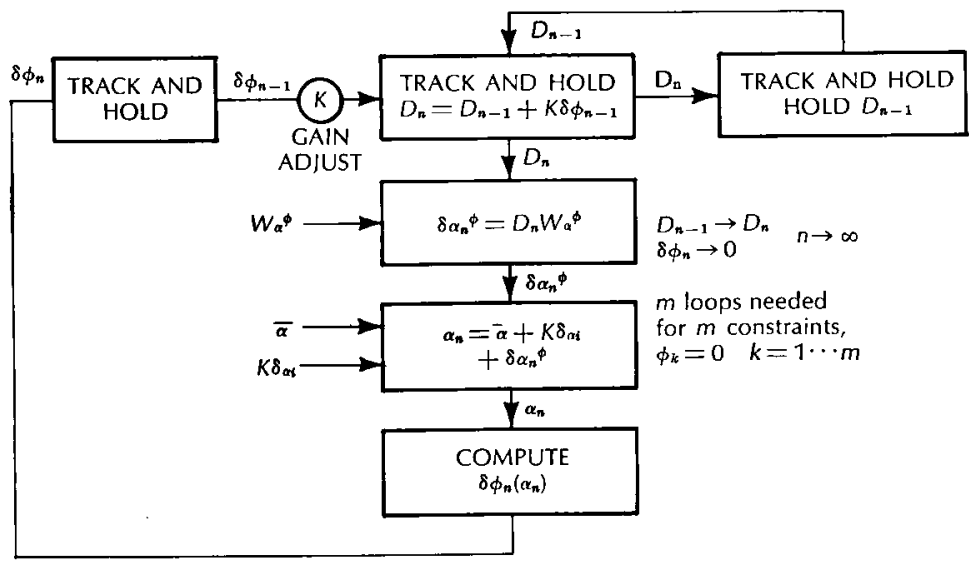

Figure 12-Iterative loop for satisfying one terminal constraint 


\section{EXAMPLE 2: SATELLITE LAUNCH TRAJECTORY OPTIMIZATION}

As an example of the use of impulse response for trajectory optimization with terminal constraints, we consider a satellite launch problem:

Determine the best program of thrust angle to the horizontal to launch a satellite into a circular orbit at prescribed altitude using minimum fuel (see figure 13).

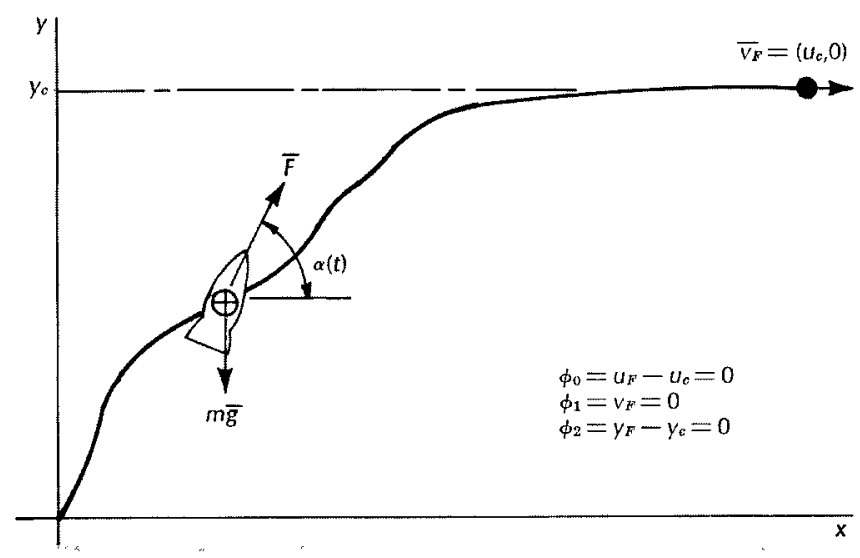

Figure 13-Satellite launch optimization problem

An analytical solution of this general problem is not known, but under the assumptions of a flat earth with constant gravity, negligible atmospheric forces and thrust magnitude proportional to a constant fuel flow rate, it is known that, for the optimal program, the tangent of the thrust angle varies linearly with time. ${ }^{5}$ Since we wished to have at least a partial analytical check of our results, the above assumptions were included in our problem. With the notation shown in figure 13, the control function, $\alpha(t)$, is the angle of the thrust to the horizontal, minimum fuel corresponds to minimum time:

$$
J=t_{F}
$$

There are three terminal constraints:

$$
\left.\begin{array}{l}
\phi_{0}=u_{F}-u_{c}=0 \\
\phi_{1}=v_{F}=0 \\
\phi_{2}=y_{F}-y_{c}=0
\end{array}\right\}
$$

We selected constants for the problem such that $u_{c}$ is the circular velocity at the orbit altitude, $y_{c}$, which was $1 / 20$ of the orbit radius. Initial lift-off acceleration was set at $1.2 \mathrm{~g}$ and maximum burning time (mass approaching zero) was 420 seconds. We selected $\phi_{0}$ as a stopping condition, and therefore had two "free" terminal constraints, $\phi_{1}$ and $\phi_{2}$, to be satisfied.

Because of the rather low initial acceleration, it is apparent that the rocket must be launched nearly vertically. We therefore chose the thrust angle program shown in figure $\mathbf{1 4}$ for the first nominal control program; the resulting trajectory is shown in figure 15. The control program slope and breakpoint were adjusted by hand until

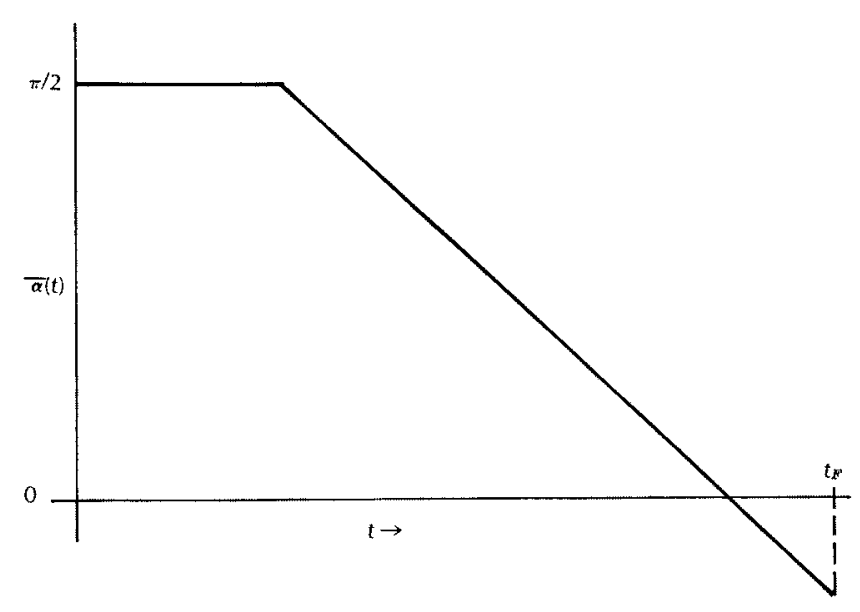

Figure 14-Initial control program

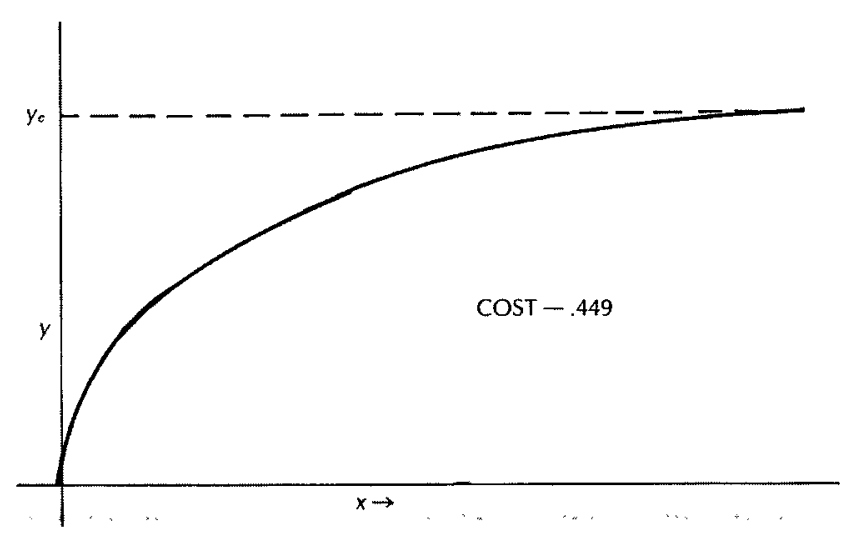

Figure 15-Initial trajectory, satellite launch

the terminal constraints were satisfied. The impulse responses of the two terminal constraints, $\phi_{1}$ and $\phi_{2}$, then were measured, simultaneously, using the procedure shown in the block diagram, figure 4 . These are shown in figures $16 \mathrm{a}$ and $16 \mathrm{~b}$ along with approximations of them which were set on diode function generators for use in the next program step.

With $W_{\alpha}^{\phi 1}$ and $W_{\alpha}^{\phi 2}$ known, the response of the cost to the control $\delta \alpha=K \delta_{\alpha i}+D_{1} W_{\alpha}^{\phi 1}+D_{2} W_{\alpha}{ }^{\phi 2}$ was measured; it is shown in figure 17. The multipliers $D_{1}$ and $D_{2}$ were adjusted automatically at each $t_{i}$ by means of two loops of the type shown in figure 12. Four iterations to adjust $D_{1}$ and $D_{2}$ were performed at each $t_{i}$ before $\delta J^{\phi}$ was plotted for that $t_{i}$ and the impulse advanced to $t_{i+1}$.

With $\delta J^{\phi}$ known, we next set an approximation of it on a hand-set diode function generator and added $K \delta J^{\phi}$ to $\bar{\alpha}$. The automatic constraint satisfaction circuitry was left in operation while the constant of proportionality was adjusted, so that the constants $D_{1}$ and $D_{2}$ were changed 


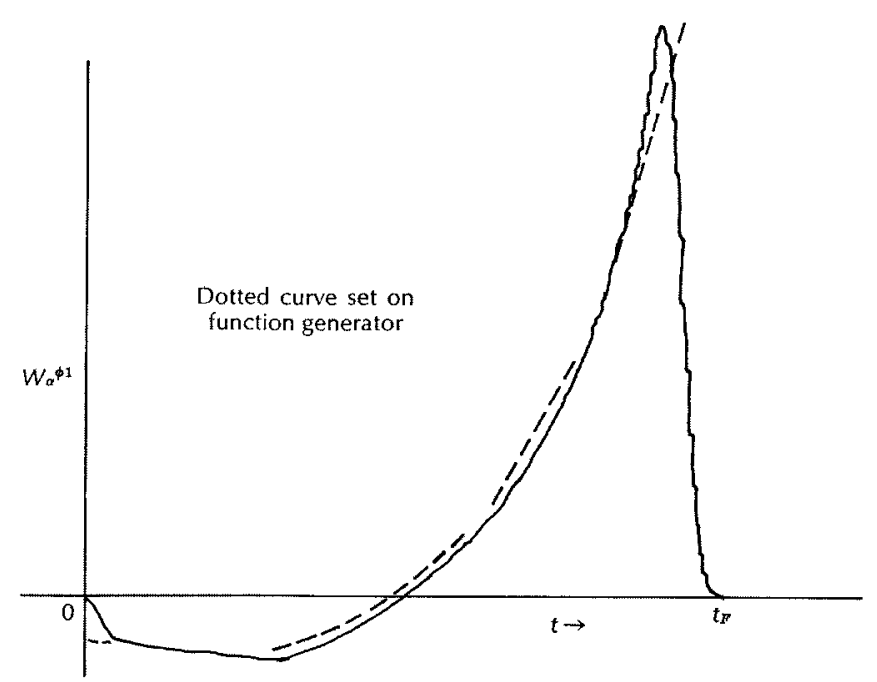

Figure $16 a-I m p u l s e$ response of terminal vertical velocity component

automatically during the adjustment of $K$. The constant $K$ was adjusted by hand until no further decrease of the cost could be noticed, and $\overline{\alpha_{1}}$ was recorded, as shown in figure 18a. Note that by leaving the constraint satisfaction circuitry in operation, the descent direction is altered auto-

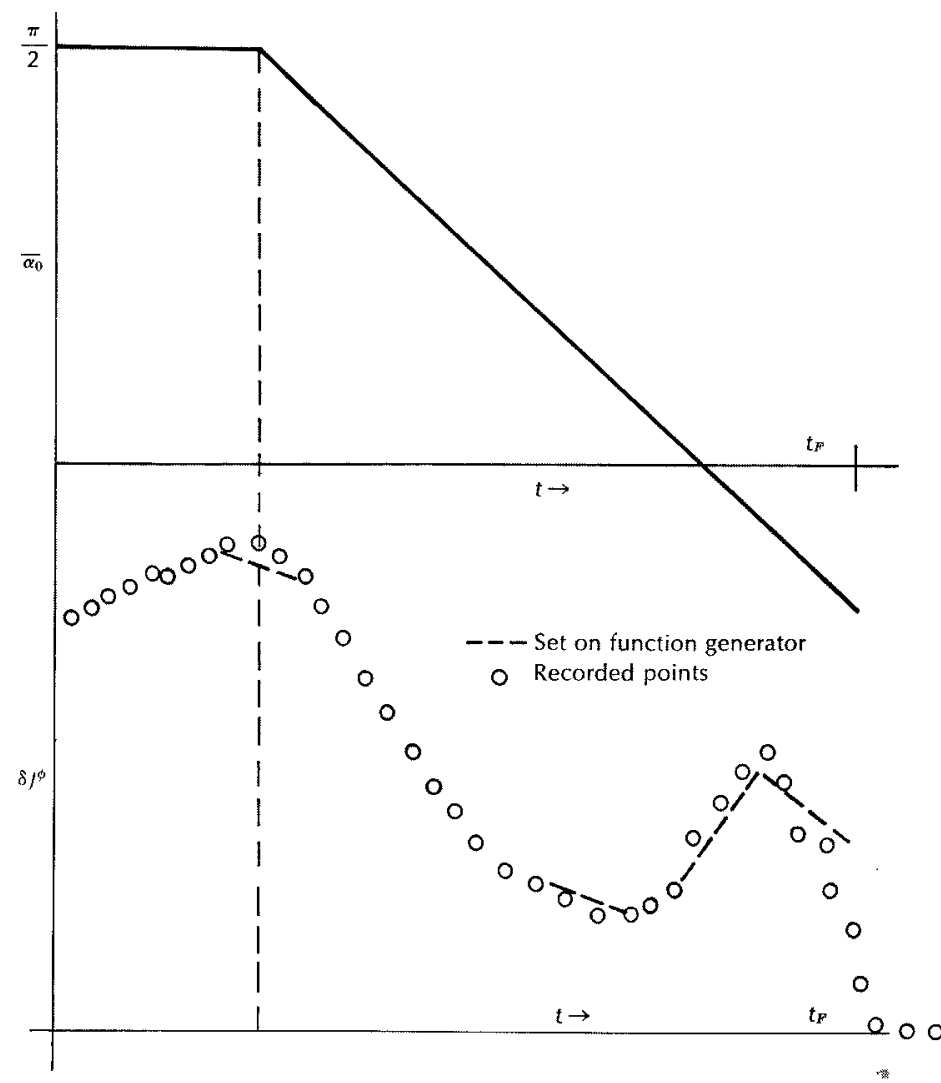

Figure 17a,b-Nominal control program and impulse response of final time, $\delta /^{\phi}$, Satellite Launch, $\phi_{1}=\phi_{2}=0$

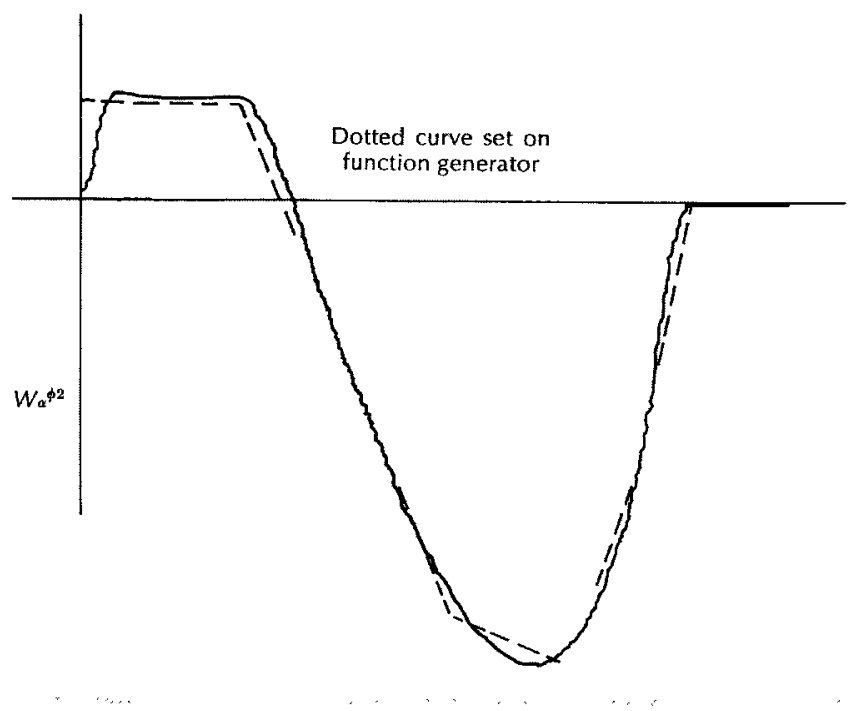

Figure 16b-Impulse response of terminal altitude

matically to account for the finite-size step. Thus no constraint error exists at the beginning of the next step. The new nominal control program, $\bar{\alpha}_{1}$, was set on a diode function generator, the gradients $W_{\alpha}^{\phi 1}$ and $W_{\alpha}{ }^{\phi 2}$ were computed again (they had changed only a small amount), and the procedure was repeated a second time. The second $\delta /^{\phi}$ is shown in figure $18 \mathrm{~b}$.

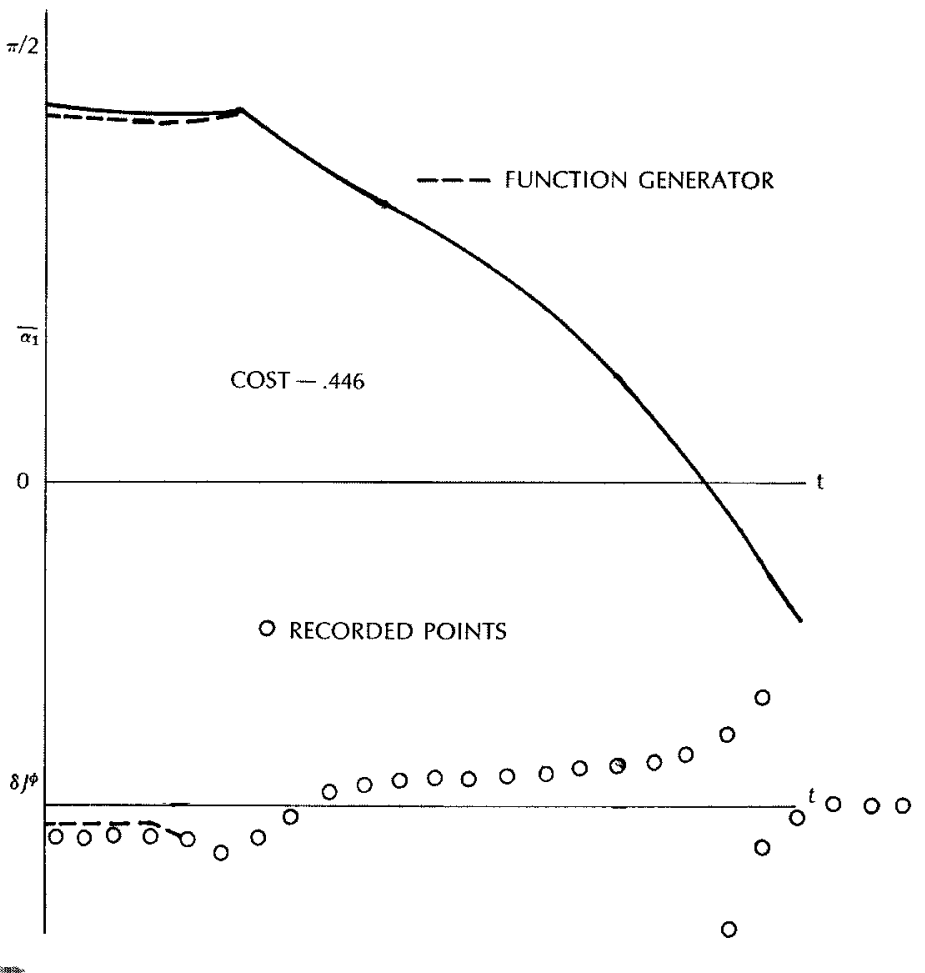

Figure 18a,b-Control program and impulse response after first iteration, Satellite Launch 


\section{DISCUSSION}

The control program resulting from only three descent steps is shown in figure 19a along with a program that has the same end points, but for which the tangent varies linearly with time, a known characteristic of the optimal program. It is apparent that we had not quite reached the optimal program, a fact confirmed by the remaining small gradient, shown in figure $19 \mathrm{~b}$.

The total decrease of cost achieved was quite small: $1 \%$ for the first iteration and about $0.1 \%$ for the second. Adding the small amount indicated by the remaining gradient shown in figure $19 \mathrm{~b}$ made a barely measurable change in total cost.

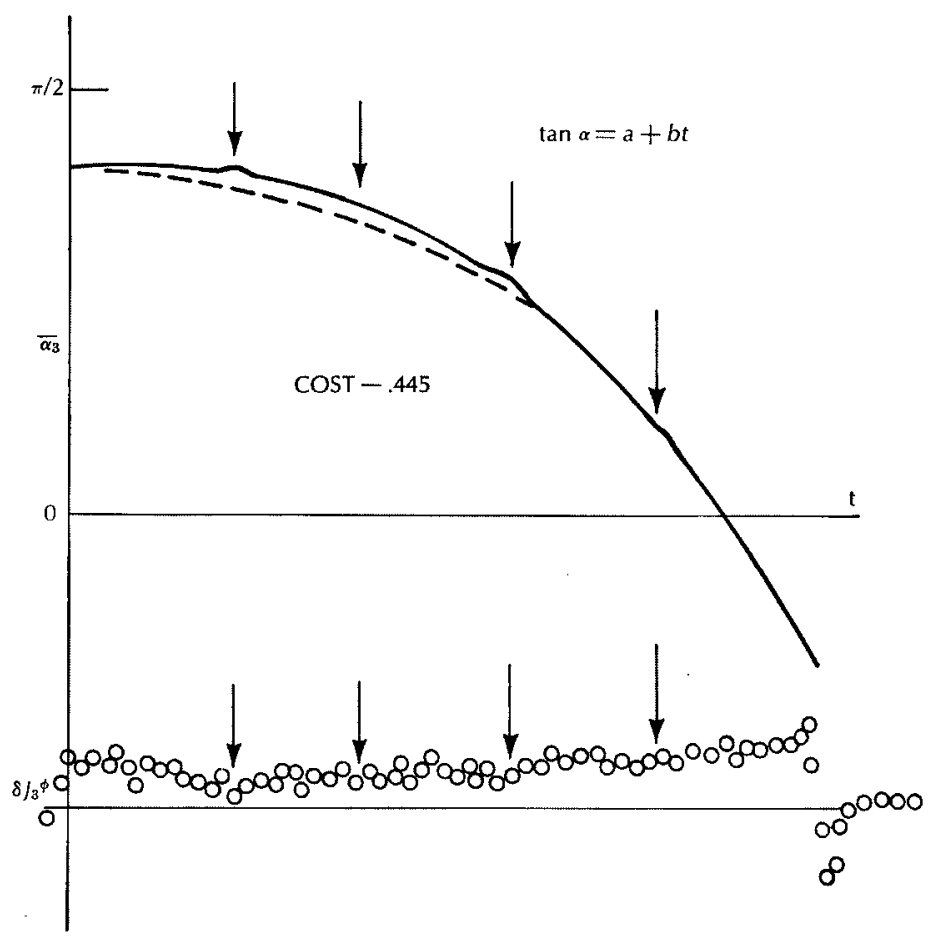

Figure 19a,b-Control program and impulse response after three iterations, Satellite Launch

The computation time required to determine a complete set of gradients was only a few seconds. Most of our time was used in adjusting the hand-set function generators and operating the plotter. If a fast hybrid computer with automatic digital function storage and generation equipment had been available, the descent to an acceptable minimum cost could have been accomplished in less than a minute, even with several terminal constraints to be satisfied.

As an indication of the sensitivity of the method, note in figure 19 that quite small irregularities in the nominal control program were detected and would be reduced in the following iteration. The fact that they were not eliminated is due to our inaccuracy in setting the hand-set function generators.

We feel that this method has several significant advantages over the more common method using adjoint equations. It is very simple and easy to understand. The mathematical techniques involved are elementary, and the information available to the analyst can be interpreted readily.

A considerable advantage is that it is not necessary to linearize the equations of motion, which may be difficult when arbitrary nonanalytical functions are involved, the usual case.

Impulse response of any number of terminal quantities can be computed, all simultaneously.

By using the automatic parameter adjustment method of satisfying terminal constraints, the descent direction is continually altered to account for finite-sized descent steps. Sizable descent steps can be taken and an acceptable minimum cost achieved in only a few steps. It is not difficult to check the second variation to assure that the cost is near at least a local minimum. Presumably, this would also detect the presence of sharp ridges and other types of discontinuities, although we have not demonstrated this.

A probable disadvantage, which we have not investigated, is that scaling of the nonlinear equations of motion cannot be expected to be as favorable as is that of the equations adjoint to the linearized equations of motion. Although it is not obvious that this means that the impulse response will not be as accurate as the gradient determined by the adjoint method, this point requires further investigation.

\section{AN ALTERNATIVE METHOD OF COMPUTING IMPULSE RESPONSE}

When several terminal constraints are imposed, the magnitude of the cost impulse response may be quite small and difficult to compute accurately. The following scheme, which we have not yet demonstrated, was devised to permit improved computation scaling and hence more precise determination of the gradient.

Instead of introducing the impulsive perturbation of the control directly into the differential equations of motion, we propose to introduce the equivalent step changes of the state variables which would result from the control perturbation.

The equations of motion, using the control function $\alpha=\bar{\alpha}+\delta \alpha$, are:

$$
\dot{x}_{i}=f_{i}\left(x_{1}, x_{2}, \cdots x_{n}, \bar{\alpha}+\delta \alpha, t\right)
$$

For a small control perturbation, $\delta \alpha$, we assume that the equations can be linearized in $\alpha$. Then, to first order:

$x_{i}(t)=x_{i o}+\int_{0}^{t} f_{1}\left(x_{1}, x_{2}, \cdots x_{n}, \bar{\alpha}, \tau\right) d \tau+\int_{0}^{t} \frac{\partial f_{i}}{\partial \alpha} \delta \alpha d \tau$

For an impulsive $\delta \alpha$,

$$
\begin{gathered}
\delta \alpha=K \delta\left(\tau-t_{i}\right) \\
x_{i}(t)=\bar{x}_{i}(t)+\left.K \frac{\partial f_{i}}{\partial \alpha}\right|_{t=t_{i}} u\left(t-t_{i}\right)
\end{gathered}
$$

where

$$
\overline{x_{i}}(t)=x_{i o}+\int_{0}^{t} f_{i}\left(x_{1}, x_{2}, \cdots x_{n}, \bar{\alpha}, \tau\right) d \tau
$$

and $u\left(t-t_{i}\right)$ is a unit step occurring at $t=t_{i}$. 
The effect of an impulsive control perturbation is synthesized by making the step changes of state variables indicated by equation (34). By using this alternative method, it should be possible to compute the effect of quite sizable control perturbations without encountering problems due to nonlinearity with respect to the control function.

\section{CONCLUSIONS}

On the basis of our limited experience, we believe that direct computation of impulse response shows great promise as a simple, fast, and accurate method of trajectory optimization. Imposition of several terminal constraints reduced the cost impulse response significantly and increased required computing time, but did not cause other computation difficulties in the cases we have investigated. Rapid convergence to a practically minimum cost has been obtained in all cases investigated so far.

An alternative direct method of computing impulse response is proposed. This method should permit better computation scaling and more accurate computation of the gradient.

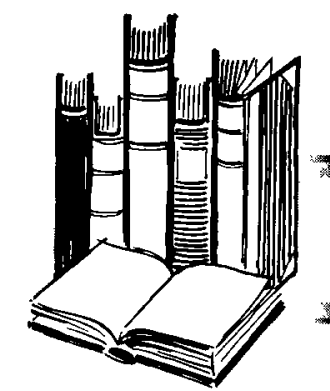

Simulation survey and literature review...

\section{REFERENCES}

1 KELLEY H J

Cradient theory of optimal flight paths

Presented at ARS semi-annual meeting Los Angeles California May 9-12 1960 ARS Journal vol 30 no 10 October 1960

also see

KELLEY H J

Method of gradients

Optimization techniques chapter 6 edited by G Leitmann Academic Press 1961

2 BRYSONAE CARROLLFJ MIKAMIK DENHAMWF Lift or drag programs that minimize re-entry heating Journal Aerospace Science vol 29 no 4 April 1962 also see BRYSON A E DENHAM W F

A steepest-ascent method for solving optimum programming problems

Raytheon Company Missile and Space Division Bedford Massachusetts Report no BR-1303 August 1961

3 WINGROVER C RABY IS

Trajectory optimization using fast-time repetitive computation

AFIPS Conference Proceedings vol 291966

Fall Joint Computer Conference November 7-10 1966

San Francisco California

4 GILBERT E G

A selected bibliography on parameter optimization methods suitable for hybrid computation SIMULATION vol 8 no 6 June 1967

5 LAWDEN D F

Interplanetary rocket trajectories

Advances in space science Academic Press 1959

\section{ANALOG SIMULATION}

1 H SCHMID (General Electric Co) Electronic analog switching

Electro-Technology p 35 June 1968

An informed and comprehensive review. Electronic analog switches have the advantage of high speed, as fast as $10 \mathrm{ns,}$ and are smaller, lighter, and more reliable. This paper features extensive discussion and illustrations of interest to designers.

2 PCSEN A K MUKHERJEE (Jadavpur Univ India)

$A$ variable frequency square wave generator with single time constant circuit International journal of Control p 409 May 1968

3 A R KAZBEKOV I M KRASSOV (USSR) Dynamic properties of electro-hydraulic servomechanisms with variable structure Avtomatika i Telemekhanika p 112 April 1968 In Russian

4 S A DOGANOVSKY (USSR) Concerning identification algorithms realized by parametric control systems Avtomatika i Telemekhanika p 155 April 1968 In Russian
5 T W CADMAN T G SMITH (Univ of Maryland) Learn about analog computers. 5: Circuit design Hydrocarbon Processing p 149 June 1968

6 S KOSHIKAWA KSUZUKI (Tokyo Medical \& Dental Univ) Study of osmo-regulation as a feedback system Medical \& Biological Engineering p 149 March 1968

7 N A FRIDLENDER (USSR) A method for complex simulation on nonstationary mass and heat transfer processes Translation no FID-HT-67-164 July 1967 AD-666077

8 B K CONANT (Arizona Univ) The design of a new solid state electronic iterative differential analyzer making maximum use of integrated circuits NASA-CR-94088 (PhD Thesis) 1968

9 R CARPENTER J ROMAN (NASA Edwards California) FM handling and analog-to-digital conversion of biomedical data from a 1,000-flight study NASA-TN-D-4488 April 1968 